\title{
Enhanced cytostatic activity of statins in mouse mammary carcinoma cells overexpressing B2-chimaerin
}

\author{
PABLO LORENZANO MENNA, ROMINA L. PARERA ${ }^{1}$, GEORGINA A. CARDAMA, \\ DANIEL F. ALONSO, DANIEL E. GOMEZ and HERNAN G. FARINA \\ Laboratory of Molecular Oncology, Department of Science and Technology, \\ Quilmes National University, Buenos Aires, Argentina
}

Received July 30, 2008; Accepted October 22, 2008

DOI: $10.3892 / \mathrm{mmr} 00000068$

\begin{abstract}
The statins, a family of cholesterol-lowering drugs, are known to block the formation of isoprenoids. They thus affect the small GTPase Rho, which requires attachment to cell membranes for proper signaling activity. Chimaerins are GTPase-activating proteins (GAPs) that accelerate GTP hydrolysis from Rac, another GTPase of the same family. We explored the cooperative antitumor effects of the overexpression of 32 -chimaerin in combination with statins. F3II mouse mammary carcinoma cells transfected with the 32-chimaerin GAP domain exhibiting low intracellular levels of active Rac-GTP were exposed in vitro to a panel of statins. Transfectants were significantly more sensitive to the cytostatic effects of lovastatin, simvastatin, atorvastatin and rosuvastatin than untransfected F3II cells with high Rac-GTP levels. Transfected tumor cells also showed a higher sensitivity for detachment from the substrate and for apoptosis after statin exposure. We further checked the cytostatic effect of statins in combination with azathioprine, a compound that specifically blocks Rac1 activation. Combined treatment with simvastatin and azathioprine demonstrated an enhanced growth-inhibitory effect on control F3II cells. Our data suggest that the combination of statins with a reduction in active Rac levels can produce a cooperative antitumor effect on breast cancer cells.
\end{abstract}

\section{Introduction}

Small GTPases of the Ras superfamily are proteins of approximately $20 \mathrm{kDa}$ that function as molecular switches for intracellular signaling pathways. They fluctuate between inactive and active states, two conformations that respectively depend on the binding of either GDP or GTP to the GTPases,

Correspondence to: Dr Daniel Gomez, Departamento de Ciencia y Tecnología, Universidad Nacional de Quilmes, R. Sáenz Peña 352, Bernal B1876BXD Buenos Aires, Argentina

E-mail: degomez@unq.edu.ar

Present Address: ${ }^{1}$ National Institute of Medicaments (INAME), Buenos Aires, Argentina

Key words: Rho GTPases, Rac, lovastatin, simvastatin, treatment, breast cancer
$(1,2)$. The switch between the active and inactive states of GTPase is highly regulated by guanine exchange factors (GEFs), which activate the GTPases by increasing the release rate of bound nucleotides, GTPase-activating proteins (GAPs) that stimulate the endogenous activity of GTPase, and GDPdissociation inhibitors that prevent the replacement of GDP by GTP (3). To date, more than 20 Rho-GTPases have been identified in humans. These can be divided into six subfamilies: Rho, Rac, Cdc42, Rnd, RhoBTB and RhoT/Miro (4).

Studies from several laboratories have established the existence of novel receptors for the forbol esters, such us chimaerins (5). Interestingly, chimaerins have Rac-GAP activity, leading to the acceleration of GTP hydrolysis from Rac1 and its subse-quent inactivation (6). In a previous report, we demonstrated that 32 -chimaerin is a key regulator of invasion and metastasis in an aggressive breast cancer model in mice (7). In that study, we showed that overexpression of the 32 -chimaerin GAP domain (B-GAP) caused alterations in actin polymerization, decreased growth rate and migratory capacity, and markedly reduced tumor invasive capacity and metastatic dissemination (7).

Rho proteins have lipid modifications at their carboxyl termini, creating a lipidated hydrophobic domain that mediates attachment to specific proteins as well as to cell membranes. Statins are cholesterol-lowering drugs that inhibit 3-hydroxy3-methylglutaryl coenzyme A (HMG-CoA) reductase, a key enzyme of the mevalonate biosynthetic pathway (8). By inhibiting the synthesis of mevalonate, statins block the formation of downstream isoprenoids, farnesyl pyrophosphate and geranylgeranyl pyrophosphate. In this regard, inhibition of the geranylgeranylation of Rho proteins appears to be an important antitumor mechanism of statins (9). Statins also inhibit the proteasome degradation machinery, leading to apoptosis and the inhibition of proliferation (10). Furthermore, we have demonstrated a dramatic impairment in the metastatic ability of lovastatin-treated mammary carcinoma cells (11).

In the present study, we explored the cooperative antitumor effects of the overexpression of B2-chimaerin in combination with statin agents, focusing on cytostatic activity and apoptosis. We used F3II mouse mammary carcinoma cells transfected with B-GAP showing low intracellular levels of Rac-GTP that were exposed in vitro to a panel of statins, and further investigated the cytostatic effect of a statin in combination with azathioprine, a compound that specifically blocks Rac1 
activation, using untransfected F3II cells with high Rac-GTP levels.

\section{Materials and methods}

Tumor cell lines and culture conditions. The mammary carcinoma cell line F3II is a highly invasive and metastatic variant, established from a clone of a spontaneous hormoneindependent Balb/c mouse mammary tumor (12). The generation of the F3II-GB1 cell line, which overexpresses B-GAP, has previously been described in detail (7). Briefly, a mammalian expression vector for $B-G A P(p C R 3 \varepsilon)$ was transfected into semiconfluent F3II mammary carcinoma cells using Lipofectamine. After 30 days of culture, colonies resistant to geneticin (Life Technologies, CA, USA) were selected by limiting dilution. Geneticin-resistant clones were then expanded, and expression of the B-GAP domain was determined by quantitative RT-PCR (7). One of the clones with high expression of $\beta-G A P$, originally named GB1, was selected for further experiments. F3II-GB1 cells and control untransfected F3II cells were cultured in MEM 41500 medium (Life Technologies) supplemented with heat-inactivated 10\% fetal bovine serum (FBS), $2 \mathrm{mM}$ glutamine and $80 \mu \mathrm{g} / \mathrm{ml}$ gentamicin at $37^{\circ} \mathrm{C}$ in a $5 \% \mathrm{CO}_{2}$ atmosphere. F3II-GB1 cells were maintained in the presence of $200 \mu \mathrm{g} / \mathrm{ml}$ geneticin. Stock cell cultures were routinely subcultured twice a week by trypsinization using standard procedures. Quantification of cell number was made by hemocytometer counting. In all cases, viability was $>90 \%$ as assayed by the trypan blue exclusion technique.

Compounds. The cholesterol-lowering drugs lovastatin, simvastatin, atorvastatin, rosuvastatin and pravastatin were kind gifts from Romikin (Buenos Aires, Argentina), while azathioprine and 6-mercaptopurin-riboside (6-MPR) were provided by Sigma Chemical Co. (St. Louis, MO, USA). The compounds were dissolved in dimethyl sulfoxide and then resuspended in MEM at a final dimethyl sulfoxide dilution of $0.5 \%(\mathrm{v} / \mathrm{v})$, a concentration which has no effect on tumor cells.

Tumor cell morphology. To examine the effect of statins on cell morphology, F3II and F3II-GB1 monolayers were cultured in 6-well plates for $24 \mathrm{~h}$ in the presence of the compounds and photographed using a phase contrast microscope (Nikon, Tokyo, Japan).

Cell proliferation assay. Cell proliferation was determined based on the conversion of MTS tetrazolium salt (Sigma) to aqueous soluble formazan product by metabolically-active cells. F3II and F3II-GB1 cells were plated at a density of $5 \times 10^{3}$ cells/well in 96-well plates, then treated with various concentrations of statins for $72 \mathrm{~h}$. At the end of the treatment period, $40 \mu 1$ of MTS reagent was added to each well. The plates were then incubated for $1 \mathrm{~h}$ at $37^{\circ} \mathrm{C}$ in the dark. The absorbance at $490 \mathrm{~nm}$ of each well was measured using an Asys UVM340 Microplate Reader (Cambridge, UK). The concentration producing $50 \%$ inhibition $\left(\mathrm{IC}_{50}\right)$ was determined by plotting the percentage of cell growth versus drug concentration. Drug combination experiments were performed under similar conditions, and cell viability was determined using the MTT metabolic assay (Sigma).
Apoptosis assay. Apoptosis was determined by a morphologic assay using ethidium bromide/acridine orange staining (EB/AO) as previously described by Coligan et al (13). Briefly, F3II and F3II-GB1 cells grown on glass coverslips were incubated with compounds for $24 \mathrm{~h}$. After treatment, cells were stained with EB/AO $(100 \mu \mathrm{g} / \mathrm{ml})$. Cells were viewed and counted using a Nikon eclipse TS100 inverted microscope at x400 magnification. Images were captured with a Nikon Coolpix digital camera. Tests were conducted in triplicate, and a minimum of 100 total cells was counted in each.

Racl activity assay and Western blotting. Tumor cells were plated at a density of $5 \times 10^{5}$ cells/well in a 6-well tissue culture plate and maintained in MEM supplemented with 10\% FBS for $24 \mathrm{~h}$. After $24 \mathrm{~h}$ in serum-free conditions, cells were stimulated with $100 \mathrm{ng} / \mathrm{ml}$ of epidermal growth factor (EGF) from Life Technologies for $15 \mathrm{~min}$. Monolayers were washed extensively with PBS and lysed in GPLB-150 buffer (20 mM Tris, pH 7.4, $150 \mathrm{nM} \mathrm{NaCl}, 5 \mathrm{mM} \mathrm{MgCl}, 0.5 \% \mathrm{NP} 40,10 \%$ glycerol, $\mathrm{pH}$ 7.4) supplemented with a protease inhibitor cocktail (Sigma). Lysates were clarified by centrifugation at $14,000 \mathrm{rpm}$ for $10 \mathrm{~min}$ at $4^{\circ} \mathrm{C}$. An aliquot was removed for the determination of total quantities of Rac1. Clarified lysates were then incubated for $1 \mathrm{~h}$ at $4^{\circ} \mathrm{C}$ with glutathione $s$-transferase-PAK-binding domain fusion protein. Fusion proteins were pre-coupled to Glutathione-Sepharose 4B beads (Amersham Biosciences, Uppsala, Sweden), and bound complexes were washed three times in GPLB-150 lysis buffer. Total cell lysates and pull-down samples were boiled for 5 min in $4 \mathrm{X}$ sample buffer and resolved by $12 \%$ SDS-PAGE. Samples were then transferred to PVDF membranes (Hybond $\mathrm{P}$, Amersham Biosciences) and analyzed by Western blotting using a monoclonal $\alpha$ Rac 1 antibody (Sigma). Antibody binding was detected by enhanced chemiluminescence.

\section{Results}

Cytostatic effect of statins on mammary carcinoma cells overexpressing $\beta-G A P$. To investigate the effect of statin agents on the proliferation of mammary carcinoma cells with high or low levels of Rac-GTP, we respectively treated control F3II cells or F3II-GB1 cells overexpressing B-GAP with five different statins (lovastatin, simvastatin, atorvastatin, rosuvastatin and pravastatin). With the exception of pravastatin, treatment with statins caused growth inhibition in both cell lines. Notably, F3II-GB1 cells appeared to be more sensitive to statin treatment than F3II cells, exhibiting significantly lower $\mathrm{IC}_{50}$ values (Table I). The effect was also observed in a dose dependent manner.

Effect of lovastatin on morphology of mammary carcinoma cells overexpressing $\beta$-GAP. We previously reported that lovastatin induced a rounded-cell morphology in F3II cells at non-cytotoxic concentrations of at least $10 \mu \mathrm{M}$, associated with the inhibition of tumor cell attachment and migration (14). Here, we evaluated the effect of statins on F3II and F3II-GB1 cell monolayers. As shown in Fig. 1, treatment with lovastatin and simvastatin at $5 \mu \mathrm{M}$ induced a rounded-cell morphology in F3II-GB1 cells, whereas no relevant effects were observed on control F3II cells at this concentration. 
Table I. Effect of statins on the in vitro proliferation of F3II and F3II-GB1 mammary carcinoma cells.

\begin{tabular}{lccccc}
\hline & \multicolumn{5}{c}{$\mathrm{IC}_{50}(\mu \mathrm{M})^{\mathrm{a}}$} \\
\cline { 2 - 6 } Cell line & Lovastatin & Simvastatin & Atorvastatin & Rosuvastatin & Pravastatin \\
\hline F3II & 43.4 & 24.3 & 42.0 & 177.7 & $>200$ \\
F3II-GB1 & $29.5^{\mathrm{b}}$ & $13.4^{\mathrm{b}}$ & $33.4^{\mathrm{b}}$ & $76.5^{\mathrm{b}}$ & $>200$ \\
\hline
\end{tabular}

${ }^{\mathrm{a}}$ F3II and F3II-GB1 cells were plated at low density and then treated with various concentrations of statins for $72 \mathrm{~h}$. Cell proliferation was revealed using the MTS metabolic assay and the $\mathrm{IC}_{50}$ values were calculated. Values represent the $\mathrm{IC}_{50}$ of each treatment using at least six determinations from three independent experiments. ${ }^{\mathrm{p}} \mathrm{p}<0.001$; ANOVA test.

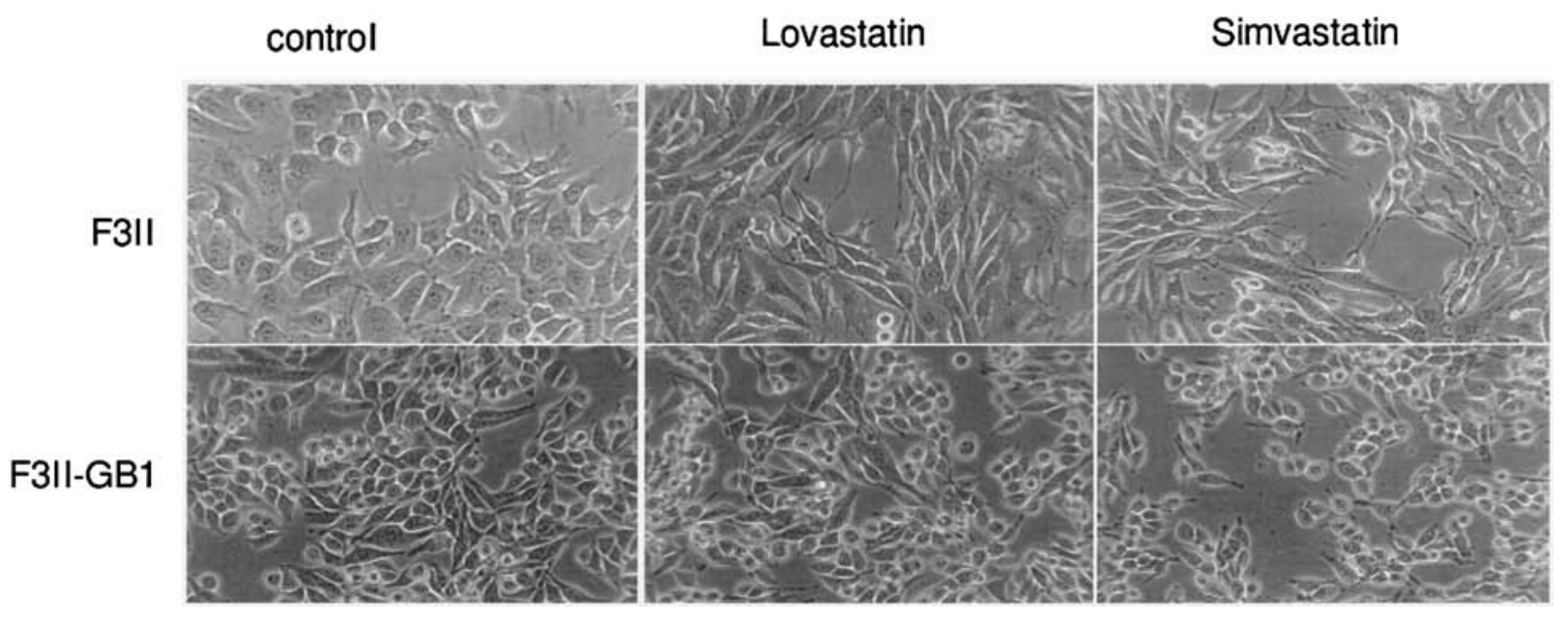

Figure 1. Effect of lovastatin and simvastatin on F3II and F3II-GB1 cell morphology. Monolayers were cultured for $24 \mathrm{~h}$ in the presence of $5 \mu \mathrm{M}$ of lovastatin and simvastatin and were then photographed using a phase contrast microscope at an original magnification of x 200 .

These data suggest that mammary carcinoma cells overexpressing ß-GAP are sensitive to statin effects on detachment from the substrate at lower doses.

Pro-apoptotic effect of simvastatin on mammary carcinoma cells overexpressing $\beta$-GAP. The pro-apoptotic effect of simvastatin was evaluated using a morphologic assay based on EB/AO staining. The compound exhibited significant proapoptotic activity in a dose-dependent manner on both F3II and F3II-GB1 cells in concentrations ranging from 5 to $20 \mu \mathrm{M}$. However, simvastatin exhibited significantly higher pro-apoptotic activity in F3II-GB1 cells compared to F3II cells at a concentration of $20 \mu \mathrm{M}$ (Table II), suggesting that mammary carcinoma cells overexpressing B-GAP have an increased sensitivity to apoptosis.

Similar effects of $\beta-G A P$ overexpression and azathioprine treatment on intracellular Rac-GTP levels of mammary carcinoma cells. We investigated the effect of B-GAP overexpression on intracellular Rac-GTP levels, as well as the effect of EGF, a well-known Rac activator. Expression of B-GAP produced a significant reduction in the basal levels of active Rac. After EGF stimuli, both control cells and transfectants showed an increase in Rac-GTP levels in comparison to their respective non-stimulated controls (Fig. 2A). We further evaluated the effect of azathioprine and a cell meta- bolite of azathioprine, 6-MPR, on Rac1 activation in F3II mammary carcinoma cells. Rac1 is the molecular target of the activity of azathioprine in T cells, as reported by Tiede et al (15). Azathioprine treatment induced an important reduction in intracellular Rac-GTP levels in control F3II cells, and 6-MPR had a stronger effect on Rac1 inhibition than the precursor drug azathioprine (Fig. 2B and C).

Table II. Pro-apoptotic effect of simvastatin on F3II and F3II-GB1 mammary carcinoma cells.

\begin{tabular}{lcc}
\hline \multirow{2}{*}{ Cell line } & \multicolumn{2}{c}{ Apoptotic cells $^{\mathrm{a}}$} \\
\cline { 2 - 3 } & Control (\%) & Simvastatin $(20 \mu \mathrm{M})(\%)$ \\
\hline F3II & 11 & $40^{\mathrm{b}}$ \\
F3II-GB1 & 12 & $58^{\mathrm{b}, \mathrm{c}}$ \\
\hline
\end{tabular}

${ }^{\text {aF } 3 I I}$ and F3II-GB1 cell monolayers were incubated for $24 \mathrm{~h}$ in the presence of simvastatin, and apoptosis was determined by EB/AO staining. Tests were conducted in triplicate, with at least 100 cells counted in each. Paclitaxel $(10 \mu \mathrm{M})$ was used as a positive control in the setup of the assay, inducing apoptosis in $\sim 80 \%$ of cells in the control monolayers. ${ }^{b} \mathrm{p}<0.001$ versus the respective control, and ${ }^{c} \mathrm{p}<0.05$ versus F3II cells; Fisher's test. 


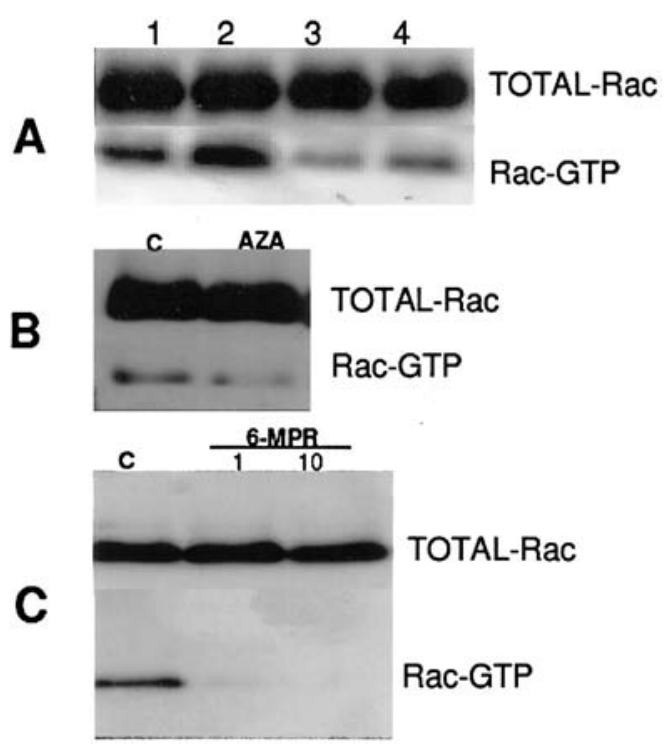

Figure 2. Pull-down Rac activity assay. (A) Control F3II (lanes 1 and 2) and F3II-GB1 cells (lanes 3 and 4). Control and transfected cells were stimulated with $100 \mathrm{ng} / \mathrm{ml} \mathrm{EGF}$ for 15 min (lanes 2 and 4, respectively). (B) F3II cells were treated for $24 \mathrm{~h}$ with $50 \mu \mathrm{M}$ of azathioprine (AZA). (C) F3II cells were treated for $24 \mathrm{~h}$ with 1 and $10 \mu \mathrm{M}$ of 6-MPR.

Increased in vitro growth inhibition of mammary carcinoma cells by a combination of simvastatin and azathioprine. Simvastatin and azathioprine treatment produced dosedependent growth inhibition in control F3II mammary carcinoma cells with respective $\mathrm{IC}_{50}$ values of $\sim 25$ and $100 \mu \mathrm{M}$ after a 72 -h exposure of log-phase growing cells. We examined the effect of a combination of simvastatin and azathioprine on this cell line. The combined treatment resulted in a significant increase in growth-inhibitory effect as compared to treatment with simvastatin or azathioprine alone (Fig. 3).

\section{Discussion}

Rho-GTPases are important intracellular signaling proteins that control diverse cellular functions related to cancer development, including gene transcription, vesicle trafficking and cytoskeleton reorganization. These processes affect the growth, differentiation, adhesion and migration of cells (16). Rho-GTPase proteins play important regulatory roles in oncogenic transfomation and in the metastatic cascade (17), emerging as useful targets for cancer treatment (18).

Several lines of evidence indicate that the Rho family member Rac may in particular play a critical role in different aspects of tumorigenesis and cancer progression. Rac was found to be an important component of Ras-induced transformation (19), and the Rac-specific GEF, Tiam1, is involved in Ras-mediated skin cancer in mice (20). Hyperactive Rac1 and Rac3 have been associated with the increased proliferation of several breast cancer cell lines $(21,22)$. In a previous study, we established that $B 2$-chimaerin, a specific Rac-GAP, is a key regulator of invasion and metastasis, and demonstrated that ectopic expression of $\beta-G A P$ cause a marked reduction in tumor invasive capacity and metastatic dissemination (7).

Statins are small-molecule inhibitors of HMG-CoA reductase that are used as cholesterol-lowering drugs. Results from

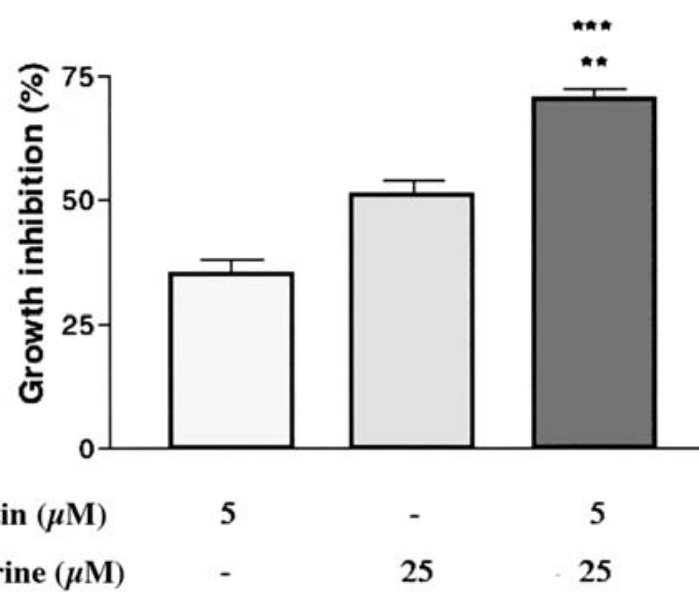

Figure 3. Combined growth inhibitory effect of simvastatin and azathioprine F3II cells were treated with simvastatin and azathioprine for $72 \mathrm{~h}$, and cell viability was measured using the MTT assay. Data were shown as the mean \pm SEM $(n=6)$, and $100 \%$ was set at the viable control cell level. Three independent experiments were performed and subjected to statistical anaysis. ${ }^{* * *} \mathrm{p}<0.001$ versus simvastatin alone; ${ }^{* *} \mathrm{p}<0.01$ versus azathioprine alone; ANOVA contrasted with the Tukey-Kramer multiple comparison test.

many pre-clinical studies have demonstrated the anticancer effect of statins on various types of cancer. Previously, we showed a dramatic impairment in the metastatic ability of lovastatin-treated F3II mammary tumor cells associated with a reduced membrane localization of Rho proteins (11).

The statin family is composed of eight unique compounds. These are naturally derived or chemically synthesized; statins derived from fungal fermentation include pravastatin, simvastatin and lovastatin, whereas fluvastatin, atorvastatin, cerivastatin, rosuvastatin and pitavastatin are synthetic compounds. The common structural characteristic of all statins is a side chain that exists in either a closed ring (inactive, lactone) or an open ring (active, acid) form. The former undergoes activation in vivo by carboxyesterases in plasma and the liver. The open ring conformation of this drug blocks catalytically-active HMG-CoA reductase by functioning as a molecular mimic of a reaction intermediate formed within the active site of this enzyme (9). Statins are effective competitive inhibitors of HMGCoA reductase - approximately 1000 -fold more effective than the natural substrate. Each member of the statin family has a similar mechanism of action, but maintains unique properties such as binding affinities, pharmacokinetics, hydrophobicity and dosage levels $(9,23)$.

In this study, we demonstrated the effects of the more representative statins used in hypercholesterolemia treatment on a cell line that overexpresses B2-chimaerin, a downmodulator of Rac activity. We found a significant decrease in cell proliferation in four of the five statins evaluated. Only pravastatin did not show an antiproliferative effect in the same dose range. Pravastatin has specific properties, such as its $\mathrm{pH}$, hydrophobicity, binding interaction and metabolic processing, that differentiate it from other statins. Pravastatin has also shown divergent results regarding growth arrest, apoptosis induction and gene expression in different cell lines. Delineating the molecular features conferring sensitivity or the lack thereof to statins is the subject of intense investigation due to the therapeutic potential they may afford $(9,24,25)$. 
An essential role in cell cycle progression has been established for Rac1. Microinjection of an activated form of Rac1 into fibroblasts stimulates DNA synthesis and cell cycle progression through $\mathrm{G} 1$, whereas a dominant-negative form of Rac1 (N17Rac1) blocks serum-induced DNA synthesis (26). Additionally, RhoA and RhoC have been found overexpressed in various human cancer types, and are associated with cell cycle progression and the increased invasiveness and metastasis of tumor cells (18). The transforming potential and cell growth stimulating activity of Rho proteins correlate with elevated cyclin D1 transcription due to its promoter activation. In this study, we explored the potential antitumoral effect of the combined inhibition of the Rho and Rac signaling pathways. We first evaluated the antiproliferative activity of five statins on F3II and F3II-GB1 tumoral cells. Our results showed a significant increase in the cell susceptibility of B-GAP-overexpressing cells to statins, suggesting a cooperative effect in the growth inhibition of two agents, with Rho and Rac small GTPases as molecular targets.

The requirement of survival signals for the prevention of apoptosis is reduced in tumoral cells. Rho proteins have been implicated in anti-apoptotic signaling and in the apoptotic process itself (27). Ectopic expression of active Rac1 can provide a survival signal to protect tumor cells or transformed fibroblasts from apoptosis (28). One proposed mechanism involves the phosphorylation of the apoptotic regulator BAD by PAK and Rho function, preventing p53-dependent apoptosis during T-cell development (29). In the present study, we demonstrated that simvastatin treatment of F3II and F3II-GB1 cells induced an increased pro-apoptotic response in F3II-GB1 transfectants.

There is a growing body of evidence to suggest that a combination of low doses of cancer therapeutic agents with varyings modes of action may produce cooperative effects on efficacy and minimize the possible side effects associated with high dose administration. Recent findings suggest that azathioprine-generated 6-Thio-GTPs inhibit the intracellular activation of Rac1 and Rac2 in T-lymphocytes, but have no effect on closely-related Rho family members such as Cdc42 and RhoA (30). We demostrated for the first time that azathioprine and its metabolite, 6-MPR, reduce the intracellular levels of active Rac in an aggressive mammary carcinoma model, and examined the antiproliferative effect of a combination of simvastatin and azathioprine on F3II cells. The combination treatment resulted in an increased growth inhibitory effect as compared to simvastatin or azathioprine alone. This result is in concordance with the differential susceptibility of F3II-GB1 and F3II to statins.

In summary, we demonstrated a cooperative effect in the growth inhibition of mammary carcinoma cells resulting from a combination of statins, drugs which affect the Rho signaling pathway by blocking the formation of isoprenoids, along with a reduction in active Rac levels due to the overexpression of chimaerins or to a specific inhibitor such as azathioprine. Our results also identify potential targets for drug combination strategies in cancer therapy.

\section{Acknowledgements}

D.F.A, D.E.G. and H.G.F. are members of the National Research Council (CONICET). This work was supported by the R\&D Grant Program 53-1004 from Quilmes National University, the Grant PIP 5101 from CONICET and by the Grant PICT 14256/03 from ANPCyT (Argentina).

\section{References}

1. Ellenbroek SI and Collard JG: Rho GTPases: functions and association with cancer. Clin Exp Metastasis 24: 657-672, 2007.

2. Buchsbaum RJ: Rho activation at a glance. J Cell Sci 120: $1149-1152,2007$

3. Rossman KL, Der CJ and Sondek J: GEF means go: turning on RHO GTPases with guanine nucleotide-exchange factors. Nat Rev Mol Cell Biol 6: 167-180, 2005.

4. Bustelo XR, Sauzeau V and Berenjeno IM: GTP-binding proteins of the Rho/Rac family: regulation, effectors and functions in vivo. Bioessays 29: 356-370, 2007.

5. Kazanietz MG: Eyes wide shut: protein kinase $\mathrm{C}$ isozymes are not the only receptors for the phorbol ester tumor promoters. Mol Carcinog 28: 5-11,2000.

6. Yang $\mathrm{C}$ and Kazanietz MG: Chimaerins: GAPs that bridge diacyl-glycerol signalling and the small G-protein Rac. Biochem J 1: 1-12, 2007

7. Menna PL, Skilton G, Leskow FC, Alonso DF, Gomez DE and Kazanietz MG: Inhibition of aggressiveness of metastatic mouse mammary carcinoma cells by the beta2-chimaerin GAP domain. Cancer Res 63: 2284-2291, 2003.

8. Konstantinopoulos PA, Karamouzis MV and Papavassiliou AG: Post-translational modifications and regulation of the RAS superfamily of GTPases as anticancer targets. Nat Rev Drug Discov 6: 541-555, 2007.

9. Wong WW, Dimitroulakos J, Minden MD and Penn LZ: HMGCoA reductase inhibitors and the malignant cell: the statin family of drugs as triggers of tumor-specific apoptosis. Leukemia 16: 508-519, 2002.

10. Rao S, Porter DC, Chen X, Herliczek T, Lowe M and Keyomarsi K: Lovastatin-mediated G1 arrest is through inhibition of the proteasome, independent of hydroxymethyl glutaryl-CoA reductase. Proc Natl Acad Sci USA 96: 7797-7802, 1999.

11. Farina HG, Bublik DR, Alonso DF and Gomez DE: Lovastatin alters cytoskeleton organization and inhibits experimental metastasis of mammary carcinoma cells. Clin Exp Metastasis 19: 551-559, 2002.

12. Alonso DF, Farias EF, Urtreger A, Ladeda V, Vidal MC and Bal de Kier Joffe E: Characterization of F3II, a mammary sarcomatoid carcinoma cell line originated from a mouse adenocarcinoma. J Surg Oncol 62: 288-297, 1996.

13. Coligan JE, Kruisbeek AM, Margulies DH, Shevach EM and Strober W: Current protocols in immunology. In: Related Isolation Procedures and Functional Assays. Vol 1. Coico R (ed). John Wiley \& Sons Inc., New York, NY, 1995.

14. Alonso DF, Farina HG, Skilton G, Gabri MR, De Lorenzo MS and Gomez DE: Reduction of mouse mammary tumor formation and metastasis by lovastatin, an inhibitor of the mevalonate pathway of cholesterol synthesis. Breast Cancer Res Treat 50: 83-93, 1998.

15. Tiede I, Fritz G, Strand S, Poppe D, Dvorsky R, Strand D, Lehr HA, Wirtz S, Becker C, Atreya R, Mudter J, Hildner K, Bartsch B, Holtmann M, Blumberg R, Walczak H, Iven H, Galle PR, Ahmadian MR and Neurath MF: CD28-dependent Rac1 activation is the molecular target of azathioprine in primary human $\mathrm{CD}^{+} \mathrm{T}$ lymphocytes. J Clin Invest 111: 1133-1145, 2003.

16. Jaffe AB and Hall A: Rho GTPases: biochemistry and biology. Annu Rev Cell Dev Biol 21: 247-269, 2005.

17. Tang Y, Olufemi L, Wang MT and Nie D: Role of Rho GTPases in breast cancer. Front Biosci 1: 759-776, 2008.

18. Sahai E and Marshall CJ: RHO-GTPases and cancer. Nat Rev Cancer 2: 133-142, 2002.

19. Zohn IE, Symons M, Chrzanowska-Wodnicka M, Westwick JK and Der CJ: Mas oncogene signaling and transformation require the small GTP-binding protein Rac. Mol Cell Biol 18: 1225-1235, 1998.

20. Malliri A, van der Kammen RA, Clark K, van der Valk M, Michiels F and Collard JG: Mice deficient in the Rac activator Tiam1 are resistant to Ras-induced skin tumours. Nature 417: 867-871, 2002.

21. Mira JP, Benard V, Groffen J, Sanders LC and Knaus UG: Endogenous, hyperactive Rac3 controls proliferation of breast cancer cells by a p21-activated kinase-dependent pathway. Proc Natl Acad Sci USA 47: 185-189, 2000. 
22. Schnelzer A, Prechtel D, Knaus U, Dehne K, Gerhard M, Graeff H, Harbeck N, Schmitt M and Lengyel E: Rac1 in human breast cancer: overexpression, mutation analysis, and characterization of a new isoform, Rac1b. Oncogene 19: 3013-3020, 2000.

23. Istvan E: Statin inhibition of HMG-CoA reductase: a 3dimensional view. Atheroscler Suppl 4: 3-8, 2003.

24. Neuvogen PJ, Backman JT and Niemi M: Pharmacokinetic comparison of the potential over-the-counter statins simvastatin, lovastatin, fluvastatin and ravastatin. Clin Pharmacokinet 47: 463-474, 2008.

25. Thelen KM, Rentsch KM, Gutteck U, Heverin M, Olin M, Andersson U, von Eckardstein A, Björkhem I and Lütjohann D: Brain cholesterol synthesis in mice is affected by high dose of simvastatin but not of pravastatin. J Pharmacol Exp Ther 316: 1146-1152, 2006.
26. Olson MF, Ashwort A and Hall A: An essential role for Rho, Rac, and Cdc42 GTPases in cell cycle progression through G1. Science 269: 1270-1272, 1995.

27. Coleman M and Olson MF: Rho GTPase signalling pathways in the morphological changes associated with apoptosis. Cell Death Differ 9: 493-504, 2002.

28. Coniglio SJ, Jou TS and Symons M: Rac1 protects epithelial cells against anoikis. J Biol Chem 276: 28113-28120, 2001.

29. Costello PS, Cleverley SC, Galandrini R, Henning SW and Cantrell DA: The GTPase rho controls a p53-dependent survival checkpoint during thymopoiesis. J Exp Med 192: 77-85, 2000.

30. Poppe D, Tiede I, Fritz G, Becker C, Bartsch B, Wirtz S, Strand D, Tanaka S, Galle PR, Bustelo XR and Neurath MF: Azathioprine suppresses ezrin-radixin-moesin-dependent T cellAPC conjugation through inhibition of Vav guanosine exchange activity on Rac proteins. J Immunol 176: 640-651, 2006. 